\title{
The Effects of after-School Training of Different Sports Events on the Physical Fitness of Adolescents and Its Comparative Analysis
}

\author{
Yanan Li' ${ }^{1}$ Alex Pak Ki Kwok ${ }^{2,3^{*}}$ \\ ${ }^{1}$ Physical Education Department, Jinan University, Zhuhai, China \\ ${ }^{2}$ School of Intelligent Systems Science and Engineering, Jinan University, Zhuhai, China \\ ${ }^{3}$ Institute of Physical Internet, Jinan University, Zhuhai, China \\ Email: ${ }^{\star}$ kwokalex@connect.hku.hk
}

How to cite this paper: Li, Y. N., \& Kwok, A. P. K. (2021). The Effects of after-School Training of Different Sports Events on the Physical Fitness of Adolescents and Its Comparative Analysis. Advances in Physical Education, 11, 321-330.

https://doi.org/10.4236/ape.2021.113027

Received: May 27, 2021

Accepted: June 27, 2021

Published: June 30, 2021

Copyright $\odot 2021$ by author(s) and Scientific Research Publishing Inc. This work is licensed under the Creative Commons Attribution International License (CC BY 4.0). http://creativecommons.org/licenses/by/4.0/

\begin{abstract}
The purpose of this study was to investigate the effects of various sports interventions on adolescents' physical fitness. One hundred twenty adolescents were recruited from a college (60 males and 60 females) and were randomly divided into four equal groups. The experimental groups (Group 1, Group 2, and Group 3) each received 1.5 hours of medium-intensity sport interventions three times a week. The only difference among them was that Group 1 did tennis, Group 2 did basketball, and Group 3 did gymnastics. Group 4, on the other hand, served as a control group, receiving no intervention. Subjects in each of the four groups were tested before and after the intervention using the National Physical Fitness Standards for Students (2014 Revision) items. The test results were analyzed using a one-way ANOVA. The independent variable was the sport natures, whereas the dependent variable was the difference between pre and post-intervention measurements. It was found that 1) the experimental groups outperformed the control group on all test indicators; 2) The basketball group improved significantly more than the tennis and gymnastics groups in terms of spirometry performance; 3 ) The gymnastics group improved significantly more than the tennis and basketball groups in terms of sitting forward bend; 4) The tennis group improved significantly more than the basketball and gymnastics groups in one-minute sit-up performance. To conclude, this study found that after-school training could promote adolescents' physical fitness. In detail, tennis training can significantly improve one-minute sit-up performance. Basketball training can significantly improve vital capacity. Gymnastic training can significantly enhance the sitting forward bend performance.
\end{abstract}




\section{Keywords}

Adolescent Health and Well-Being, Active after School Programs, Physical Fitness, Sport Interventions, School Sport

\section{Introduction}

Regular engagement in youth sport has a range of benefits (Perkins \& Hahn, 2020) and has been shown to improve important physical qualities as muscle endurance (Faigenbaum et al., 1999), coordination (Loprinzi et al., 2012), flexibility (Merkel, 2013), aerobic capacity (Boreham \& Riddoch, 2001) and muscular strength (Mohamed et al., 2009). However, systematic and comprehensive comparisons of which sports have more significant effects on which qualities are lacking. To that end, this research aimed to investigate the effect of after-school training on adolescents' physical fitness and how different sports impact the outcome. The study, described in greater detail below, provided after-school sports training (tennis, basketball, and gymnastics) to 120 Chinese students (50\% females, 50\% male). This study conducted after-school training in tennis, basketball, and gymnastics for adolescents in a college. The subjects' activities were tracked and monitored. At the end of the experiment, all subjects were required to participate in physical fitness tests. The tests were conducted following the National Physical Fitness Standards for Students (2014 Revised). The findings could aid trainers in developing a more tailored exercise program for teenagers.

\section{Research Objects and Methods}

\subsection{Ethical Considerations}

This study adhered to the American Psychological Association Code of Ethics and was approved by the Institutional Review Board of the authors' institution. Every subject was required to sign an informed consent form before the start of the experiment. 120 year-6 students were recruited from a local college.

\subsection{Overview of Approach}

The present study used a research design of 4 groups (Group 1: tennis group, Group 2: basketball group, Group 3: gymnastics group, and Group 4: control group) to provide different interventions to each of the four experimental groups. Subjects were required to complete a pre-physical fitness test at the beginning of the experiment. Once completed, Group 1, Group 2, and Group 3, which consist of 90 subjects, received different after-school training according to the experiment design at the beginning. Subjects in these three experimental groups were regularly monitored during the experiment to ensure their exercise duration, exercise frequency, and exercise intensity were reasonably consistent. In contrast, the control group (Group 4) did not receive any long-term struc- 
tured after-school training other than everyday physical exercises. At the end of the program, subjects completed the physical fitness again to make an accurate and detailed comparison.

\subsection{Experimental Variables}

\section{Independent variable:}

Nature of sports-Group 1: tennis group, Group 2: basketball group, Group 3: gymnastics group, and Group 4: control group (not participate in any sport).

\section{Dependent variable:}

Change in performance in the physical fitness test.

\subsection{Subjects}

The subjects were all year- 6 students from a local college. The experimental groups consist of students who were originally enrolled in after-school training programs. These students were randomly chosen as the study subjects and were evenly distributed into Group 1, Group 2, and Group 3. In contrast, the control group was made up of 15 male and 15 female students who were chosen at random from the unenrolled students from the college.

Experimental group:

- Group 1 (tennis program intervention group) consisted of students who voluntarily signed up for the after-school tennis instruction program (30 students; male: female $=1: 1$ ).

- Group 2 (basketball program intervention group) included students willingly signed up for the after-school basketball training program (30 students; male: female $=1: 1)$.

- Group 3 (gymnastics program intervention group) was made up of students who voluntarily sign up for the after-school gymnastics programs, including aerobics (18 students) and wushu (12 students) (30 students; male: female $=1: 1$ ).

Control group:

- Group 4 was made up of students who do not engage in any after-school training program (30 students, male: female $=1: 1)$ (Figure 1).

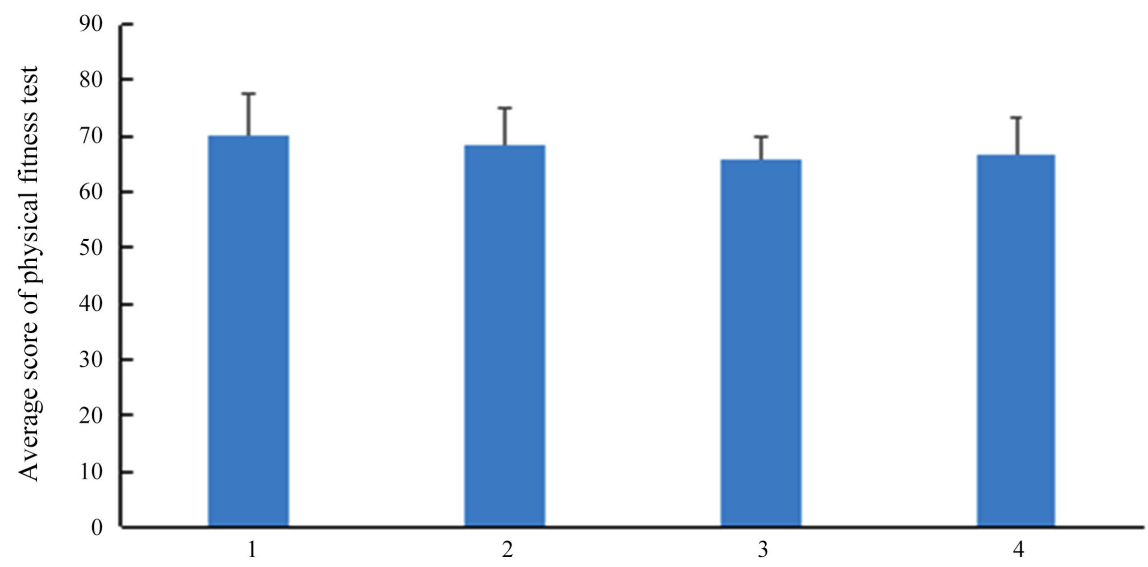

Figure 1. Average score of physical fitness test before exercise intervention. 
All subjects in this study were in good health, with no cardiovascular conditions that made them unfit for exercise, and a gender ratio of 1:1. The four groups' pre-intervention physical fitness test scores were evaluated using one-way ANOVA. As a result, no substantial difference was found among the four groups $(p>0.05)$. This outcome meant that the four groups were essentially at the same level in physical fitness scores before the exercise intervention.

\subsection{Exercise Intervention}

Program coaches guided subjects in experimental groups to attend 1.5-hour after-school training sessions three days a week (Monday, Wednesday, and Friday), including technical and physical training. In contrast, the control group did not engage in any after-school training during the experiment except for everyday exercise. The intervention lasted two semesters.

\subsection{Statistical Analysis}

The physical fitness test results were gathered, organized, and analyzed using the SPSS17.0 statistical software. Apart from the test scores, background data of the subjects, such as BMI, were also collected. Since the pre-test results may affect the post-test results, this study used the difference between the pre and post-test results as an indicator for performance comparison. One-way ANOVA was employed to analyze the difference in performance among the four groups.

\section{Experimental Results}

\subsection{Comparison of the Difference in Total Physical Fitness Test Scores between Groups before and after Exercise Intervention}

After two semesters of sports intervention, the physical fitness test scores of subjects in each group showed unequal changes, with Group $2>$ Group $1>$ Group $3>$ Group 4. One-way ANOVA was conducted to analyze the difference in physical fitness test scores before and after the intervention. As a result, a significant difference was found between the experimental group (Group 1, Group 2, and Group 3) and the control group (Group 4) (p = 0.000, 0.000, and 0.000, respectively). However, the discrepancies between the groups were not significant when compared two by two $(\mathrm{p}=1>0.05)$. This result suggested that all three programs (tennis, basketball, and gymnastics) positively impact subjects' physical fitness test scores. Besides, the growth in scores with exercise interventions is substantially greater than natural growth (Figure 2).

\subsection{Comparison of Individual Physical Fitness Test Scores before and after Exercise Intervention}

\subsubsection{Comparison of the Difference in Spirometry Scores in Each Group before and after Exercise Intervention}

Spirometry is a vital functional indicator that represents the level of growth and development of a person. It refers to the amount of gas exhaled as forcefully as 
possible by the human body after a single maximum exhalation with no time limit. It thus defines the extent of development of body functions, representing the maximum functional operation of the lung organs at one time. The spirometry score indirectly indicates a person's lung function. A person with a high vital capacity is able to deliver more oxygen to the body in a single breath. Figure 4 displays the differences between the two spirometry scores before and after the two semesters of the exercise intervention. The results revealed the impact of the exercise intervention on the subjects' spirometry test scores. A positive value indicates an improvement in subjects' spirometry test scores over the two semesters. In contrast, a negative difference value shows a reduction in spirometry test scores before and after the two semesters of study (Figure 3).

The spirometry test scores of subjects in each group showed unequal changes after two semesters of exercise intervention, with Group $2>$ Group $1>$ Group $3>$ Group 4 . The differences in their spirometry test scores before and after exercise intervention were analyzed using one-way ANOVA. The discrepancies

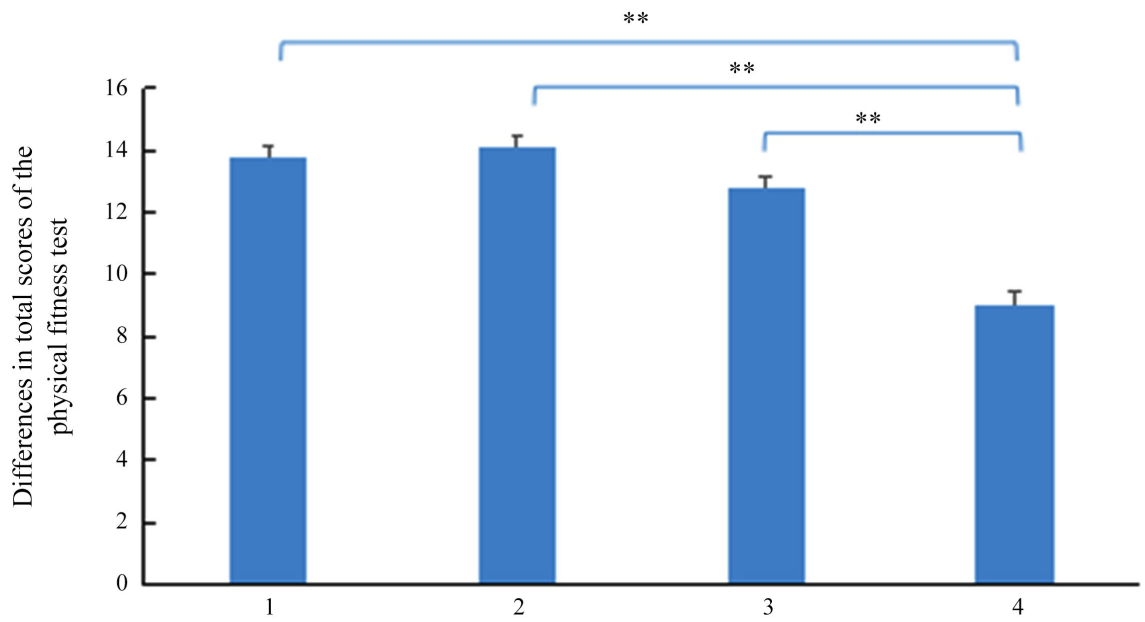

Figure 2. Differences in total scores of the physical fitness test before and after the exercise intervention. ${ }^{*}$ represents $\mathrm{p}<0.01{ }^{*}$ represents $\mathrm{p}<0.05$.

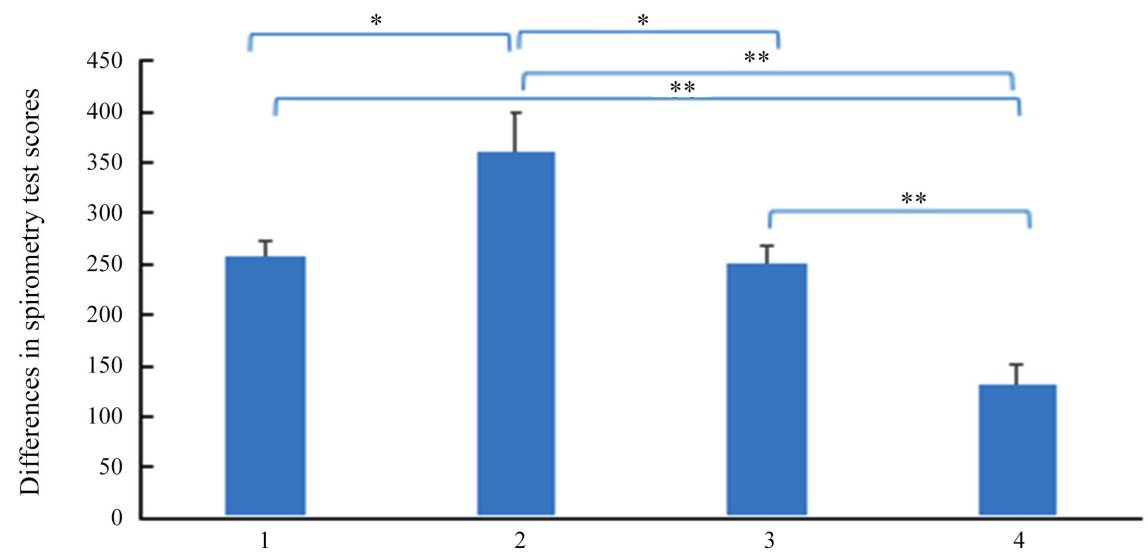

Figure 3. Differences in spirometry test scores before and after the exercise intervention. ${ }^{*}$ represents $\mathrm{p}<0.01 ;{ }^{*}$ represents $\mathrm{p}<0.05$. 
between the experimental group (Group 1, Group 2, and Group 3) and the control group (Group 4) were significant ( $\mathrm{p}=0.002,0.000$, and 0.006, respectively). This result suggested that all three exercise programs could substantially improve subjects' lung function performance. Besides, a two-by-two comparison of Group 1, Group 2, and Group 3 revealed significant differences between Group 1 and Group $2(\mathrm{p}=0.027)$, as well as between Group 2 and Group $3(\mathrm{p}=0.013)$. Alternatively, no significant difference was found between Group 1 and Group 3 $(\mathrm{p}=1.000)$. This outcome meant that the basketball program intervention improved subjects' spirometry efficiency the most, substantially more than the tennis program intervention, gymnastics program intervention, or natural increase.

\subsubsection{Comparison of the Difference between the Scores of Sitting forward Bend Test in Each Group before and after Exercise Intervention}

The seated forward bend test is often used to determine the maximum range of motion of the trunk, waist, and hip joints at rest. It reflects the stretch and elasticity of the above-mentioned joints, ligaments, and muscles. It is the most important test indicator of physical flexibility growth (Figure 4).

The subjects in each group had unequal changes in their seated forward bend test scores after two semesters of exercise intervention, with Group $3>$ Group $1>$ Group $2>$ Group 4. The differences in their seated forward bend test scores before and after the exercise intervention were analyzed using a one-way ANOVA. The differences between the experimental groups (Group 1, Group 2, and Group 3) and control group (Group 4) were substantial ( $\mathrm{p}=0.001,0.002$, and 0.000 , respectively). This result indicated that all three exercise programs could help subjects perform better on seated forward bend tests. Besides, a two-by-two analysis of Group 1, Group 2, and Group 3 showed significant differences between Group 1 and Group $3(\mathrm{p}=0.022)$ and Group 2 and Group $3(\mathrm{p}=$ $0.013)$. Alternatively, no significant difference was found between Group 1 and Group $2(\mathrm{p}=1.000)$. This result revealed that the gymnastics program intervention improved subjects' physical flexibility the most, substantially more than the tennis program intervention, basketball program intervention, or natural increase.

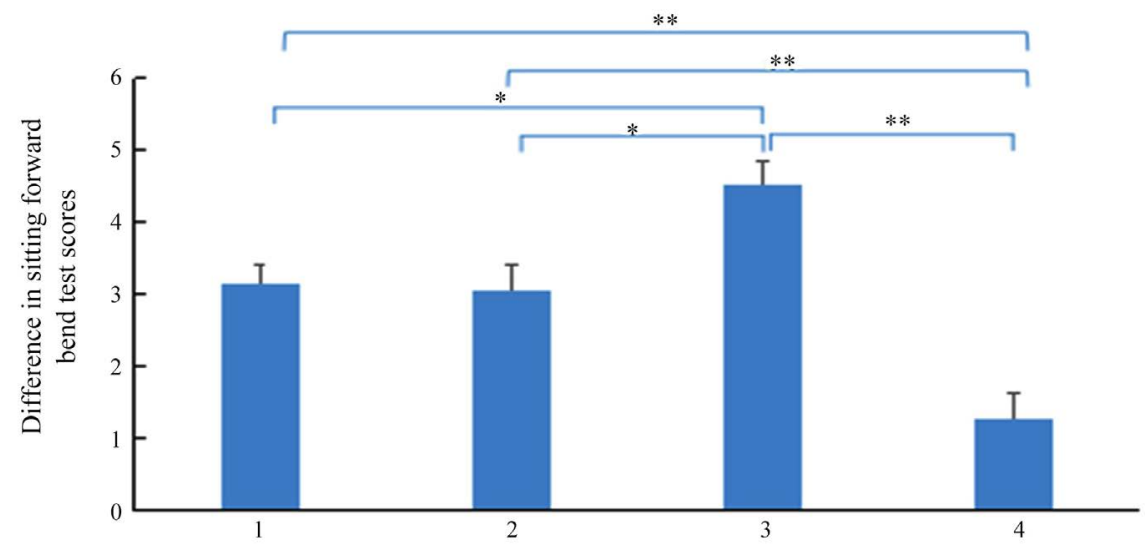

Figure 4. Difference in sitting forward bend test scores before and after the exercise intervention. ${ }^{* *}$ represents $\mathrm{p}<0.01{ }^{*}$ represents $\mathrm{p}<0.05$. 


\subsubsection{Comparison of the Difference of One-Minute Sit-up Test Scores in Each Group before and after Exercise Intervention}

The sit-up test is a fitness test that measures the strength and endurance of the muscles in the human waist and abdomen (Figure 5).

Subjects in each group reported varying improvements in their one-minute sit-up test scores after two semesters of the exercise intervention, with Group $1>$ Group $2>$ Group $3>$ Group 4. One-way ANOVA was performed to analyze the difference in one-minute sit-up test scores before and after the exercise intervention. The differences between the experimental groups (Group 1, Group 2, and Group 3) and control group (Group 4) were substantial ( $\mathrm{p}=0.000,0.015$, and 0.017 , respectively). This outcome meant that all three exercise programs could potentially improve subjects' performance on one-minute sit-up tests. Besides, a two-by-two analysis of Group 1, Group 2, and Group 3 showed significant differences between Group 1 and Group $2(\mathrm{p}=0.000)$ and Group 1 and Group $3(\mathrm{p}=0.000)$. Alternatively, no significant difference was found between Group 2 and Group 3 ( $\mathrm{p}=$ 1.000). This finding showed that the tennis program intervention increased subjects' strength and endurance of the muscles significantly more than the basketball program intervention, gymnastics program intervention, or natural increase.

\section{Discussion}

\subsection{Comparative Analysis of the Difference in Total Physical Fitness Test Scores before and after Exercise Intervention}

Subjects in all three experimental groups (Group 1, Group 2, and Group 3) substantially increased their physical fitness test scores after two semesters of exercise intervention. This outcome was because subjects in the experimental groups were more active in exercise than the control group during the experiment. Hence, based on the relationship between physical activity and physical fitness, subjects in the experimental groups should be physical "healthier" than the subjects in the control group. The exercise intervention habits in the three experimental groups improved physical fitness test scores more than natural growth.

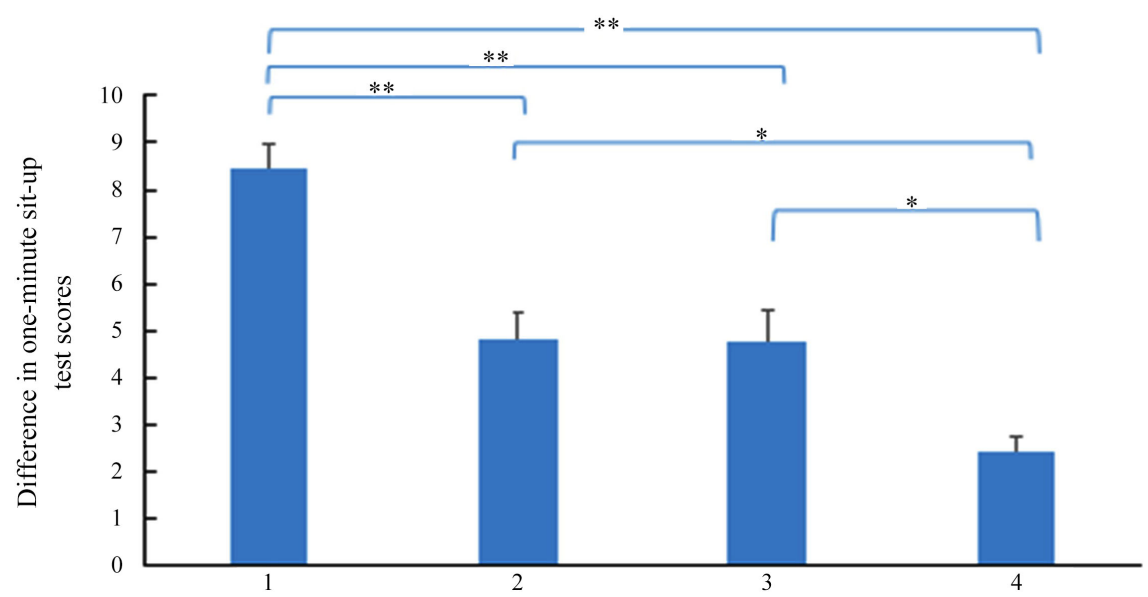

Figure 5. Difference in one-minute sit-up test scores before and after the exercise intervention. ${ }^{*}$ represents $\mathrm{p}<0.01{ }^{*}$ represents $\mathrm{p}<0.05$. 


\subsection{Comparative Analysis of Individual Test Scores of Physical Fitness Test before and after Exercise Intervention}

\subsubsection{Comparative Analysis of the Difference in Spirometry Test Scores of Each Group before and after Exercise Intervention}

Li Yongming (2013) pointed out that the rate of aerobic energy supply could hit more than $96.8 \%$ in sports activities with an exercise period of more than 10 minutes. As subjects used to have a high demand for oxygen and vital capacity during the exercises, the human body naturally increases the volume of a single gas exhalation and inhalation when stimulated for an extended period. Vital volume refers to the largest volume of gas exhaled by the human body after a single maximum inhalation. Hence, a long time of tennis, basketball, or gymnastics training undoubtedly increases the human body's vital capacity. Since the subjects who took part in the experimental groups had never received systematic instruction in the sport before, they were only at a primary competitive stage.

All three sports require relentless effort to counteract the athletes' own gravity for movement. For example, for tennis, athletes must continue to move quickly while hitting the ball. The quick movement of the ball after the stroke, primarily overcoming the athletes' own gravity, and the kinetic energy of the tennis ball acting on the racket, are the most powerful single stimulus of the tennis sport. For basketball, athletes must keep moving fast while holding the ball. In addition to dribbling, passing, and shooting motions that transcend the athletes' own gravity to travel rapidly and acting force on the basketball, the basketball sport also involves physical contact with opposition athletes from time to time. For gymnastics, athletes must continue to work out to counteract their gravity by leaping and spinning. Gymnastics is a systematic activity that allows people to act despite their own gravity. When comparing the three sports, tennis, basketball, and gymnastics should make athletes perform work out continuously during the exercise. However, in terms of single stimulus intensity, basketball athletes need to transcend their own gravity and engage in physical combat with opposing athletes. Hence, the intensity of the single stimulus received in the basketball activities should be greater than that in tennis and gymnastics. Hence, the basketball program intervention would have a more significant impact on body functions, especially oxygen demand and consumption, as well as chest expansion and respiratory system improvement. As a result, subjects in the basketball program intervention group had a greater effect on spirometry test score increases before and after the two-semester exercise intervention than subjects in the tennis program intervention group or the gymnastics program intervention group.

\subsubsection{Comparative Analysis of the Difference between the Scores of Sitting forward Bend Test in Each Group before and after the Exercise Intervention}

According to the findings, the gymnastics intervention had the greatest impact on the growth of subjects' test scores in the seated forward bend. This result was largely dictated by the gymnastics program's characteristics and training me- 
thods. Athletes need to show off their bodies as much as possible in the gymnastics sport. They often need to perform complicated movements such as splits, which necessitate a torso stretch and elasticity. Even during jumping, tumbling, and spinning, the body must also be stretched and strengthened. Because of these characteristics, gymnastics would have a significant effect on subjects' body flexibility. Although tennis and basketball also require the human body to make certain unusual movements to cope with unpredictable circumstances, the motion is rarely high. Hence, there is little training regarding body flexibility in the program. As a result, subjects in the gymnastics program intervention had a more significant impact on subjects' performance in the seated forward bend test than the tennis program and the basketball program.

\subsubsection{Comparative Analysis of the Difference between the One-Minute Sit-up Test Scores of the Groups before and after the Exercise Intervention}

One-minute sit-ups primarily respond to the waist and abdomen's power capability. It tests a person's muscle strength on the one hand and muscle endurance on the other. The strength of the waist and abdomen is the core of the whole body's strength, acting as a support in the movement process. They are essential to carry out the motions in tennis, whether it is serving or backhand pumping at the baseline. As the most important part of the power chain, the power of all motions when playing tennis cannot be separated from the role of the waist and abdomen. In contrast, when playing basketball, the body is in displacement motion most of the time. Even in technical motions such as passing, catching, and throwing, the role of the waist and abdomen mobilized is far from being comparable to the role in tennis. Despite the fact that the waist and abdomen are often mobilized during physical confrontations, the frequency of mobilization per unit of time in the entire training or game cannot be comparable to tennis. Lastly, gymnastics is a sport that performs motions by overcoming athletes' own gravity. Although the frequency of mobilization of the waist and abdomen per unit of time is comparable to that of a tennis program, the capacity to do work is vastly different. In contrast, tennis requires athletes to overcome gravity for movement and transfer the force generated by the lower limb stomp to the racket holding hand to hit the tennis ball. Therefore, the power and energy of the work done by the lumbar abdomen mobilized by tennis exceed basketball and gymnastics, both in training and during competition. As a result, subjects in the tennis program intervention group had a greater impact on the increase in one-minute sit-up program test scores than the basketball program intervention group and the gymnastics program intervention group.

\section{Conclusion}

This study set out to determine the effects of various sports interventions on adolescents' physical fitness. 120 year-6 students were recruited from a local college. Our findings suggest that 1) after-school training in different sports could 
significantly improve the physical fitness of adolescents; 2) after-school training has specific characteristics in promoting the physical fitness of adolescents. In summary, tennis training can improve one-minute sit-up performance significantly. Basketball training can improve vital capacity significantly. Gymnastics training can significantly enhance sitting forward bend performance. Further research should be performed to other age groups and sports.

\section{Acknowledgements}

This work was supported by the National Natural Science Foundation of China [grant numbers 72001091]; Guangdong Basic and Applied Basic Research Foundation [grant numbers 2020A1515110889]; and Fundamental Research Funds for the Central Universities of China [grant numbers 21619320].

\section{Conflicts of Interest}

The authors declare no conflicts of interest regarding the publication of this paper.

\section{References}

Boreham, C., \& Riddoch, C. (2001). The Physical Activity, Fitness and Health of Children. Journal of Sports Sciences, 19, 915-929. https://doi.org/10.1080/026404101317108426

Faigenbaum, A. D., Westcott, W. L., Loud, R. L., \& Long, C. (1999). The Effects of Different Resistance Training Protocols on Muscular Strength and Endurance Development in Children. Pediatrics, 104, Article No. e5. https://doi.org/10.1542/peds.104.1.e5

Li, Y. (2013). Energy Metabolism of Different Exercise Modes: Common and Difference. China Sport Science, 33, 81-86.

Loprinzi, P. D., Cardinal, B. J., Loprinzi, K. L., \& Lee, H. (2012). Benefits and Environmental Determinants of Physical Activity in Children and Adolescents. Obesity Facts, 5, 597-610. https://doi.org/10.1159/000342684

Merkel, D. L. (2013). Youth Sport: Positive and Negative Impact on Young Athletes. Open Access Journal of Sports Medicine, 4, 151-160. https://doi.org/10.2147/OAJSM.S33556

Mohamed, H., Vaeyens, R., Matthys, S., Multael, M., Lefevre, J., Lenoir, M., \& Philippaerts, R. (2009). Anthropometric and Performance Measures for the Development of a Talent Detection and Identification Model in Youth Handball. Journal of Sports Sciences, 27, 257-266. https://doi.org/10.1080/02640410802482417

Perkins, P., \& Hahn, A. (2020). Considerations and Suggestions for Design of a Learning and Development Program for Sport Coaches. Open Journal of Social Sciences, 8, 457-509. https://doi.org/10.4236/jss.2020.812036 